\title{
1 Clinicians' management of patients potentially exposed to rabies in high-risk areas in 2 Bhutan: A cross-sectional study
}

3 Kinley Penjor ${ }^{1,2,3^{*} \sharp a}$, Nelly Marquetoux ${ }^{1}$, Chendu Dorji1 ${ }^{1,2,4}$, Kinley Penjor ${ }^{1,2,5}$, Sithar Dorjee ${ }^{1,5}$, Chencho

$4 \quad$ Dorjee $^{7}$, Peter D Jolly ${ }^{1}$, Roger S Morris ${ }^{6}$ and Joanna S. McKenzie ${ }^{1}$

${ }^{1}$ School of Veterinary Science, Massey University, New Zealand.

${ }^{2}$ Khesar Gyalpo University of Medical Sciences of Bhutan, Thimphu, Bhutan.

${ }^{3}$ Sarpang District Hospital, Department of Medical Services, Ministry of Health, Thimphu Bhutan.

${ }^{4}$ Regional Livestock Development Centre Tsimasham, Department of Livetsock, Ministry of Agriculture and Forest

$11{ }^{6}$ Morvet Ltd, Consultancy services in health risk management and food safety policy and programs, Masterton, New

12 Zealand.

${ }^{7}$ Faculty of Nursing and Public Health, Khesar Gyalpo University of Medical Sciences of Bhutan, Thimphu, Bhutan.

${ }^{\# a}$ Current address: Dewathang Military Hospital, Samdrup Jongkhar, Department of Medical services, Ministry of 


\section{Abstract}

23 Background: Rabies is endemic in southern Bhutan, associated with 1-2 human deaths annually and

24 accounting for about $6 \%$ of annual national expenditure on essential medicines. A WHO-adapted

25 National Rabies Management Guidelines (NRMG) is available to aid clinicians in PEP prescription. An

26 understanding of clinical practice in the evaluation of rabies risk in endemic areas could contribute to

27 improve clinicians' PEP decision-making.

Methods: A cross-sectional survey of clinicians was conducted in 13 health centers in high-rabies-risk

29 areas of Bhutan during February-March 2016. Data were collected from 273 patients examined by 50

30 clinicians.

31 Results: The majority (69\%) of exposure was through dog bites. Half the patients were children under 18

32 years of age. Consultations were conducted by health assistants or clinical officers (55\%), or by medical

33 doctors (45\%), with a median age of clinicians of 31 years. Rabies vaccines were prescribed in $91 \%$ of

34 exposure cases. The overall agreement between clinician's rabies risk assessment and the NRMG for the

35 corresponding exposure was low (kappa $=0.203, p<0.001$ ). Clinicians were more likely to underestimate

36 the risk of exposure than overestimate it. Male health assistants were the most likely to make an

37 accurate risk assessment and female health assistants were the least likely. Clinicians from district or

38 regional hospitals were more likely to conduct accurate risk assessments compared to clinicians in Basic

39 Health Units (Odds Ratios of 7.8 and 17.6, respectively).

40 Conclusions: This study highlighted significant discrepancies between clinical practice and guideline

41 recommendations for rabies risk evaluation. Regular training about rabies risk assessment and PEP

42 prescription should target all categories of clinicians. An update of the NRMG with more specific

43 criterions for the prescription of RIG might contribute to increase the compliance, along with a regular

44 review of decision-making criteria to monitor adherence to the NRMG. 


\section{Author summary}

46 Human rabies remains an important public health threat in Bhutan, especially in southern regions where

47 canine rabies is endemic. The steady increase in number of patients reporting to hospitals following dog

48 bites means escalating costs of post-exposure prophylaxis for the country. We investigated attitudes and

49 practices of clinicians who manage patients with potential rabies exposure, in the endemic area. The risk

50 of rabies exposure in the study area is mostly associated with dog bites, involving children half the time.

51 Rabies vaccines were prescribed in 9 out of 10 exposure cases, while immuno-globulins were rarely

52 prescribed. The study confirmed the perceived lack of compliance of clinicians with guideline

53 recommendations for assessing rabies risk. This results in under-estimating the rabies risk in potentially

54 risky exposures in high-rabies-risk areas. Our work underscore the importance of targeted training of

55 female health assistants, doctors, and clinicians in basic health units to improve the management of

56 rabies exposure. In particular there is need to update the national guidelines regarding indications and

57 use of rabies immune-globulins.

\section{Introduction}

59 In Asia, rabies remains a major public health threat, with an estimated 39,000 deaths annually, mostly

60 due to spill over from the canine reservoir [1]. Wider use of post-exposure prophylaxis (PEP) might

61 reduce human mortalities in this region of the world [2]. On the other hand, the escalating cost of life-

62 saving PEP represents a major burden to both national economies and families, mostly in poor rural

63 communities $[1,3,4]$.

64 In Bhutan, the number of reported animal rabies cases was stable in the decade 1996 to 2005 but

65 increased during 2006 to 2008, mostly in 4 districts in southern Bhutan bordering India [5]. Maintenance

66 of rabies in the canine reservoir in the south Bhutan was likely due to low coverage of dog vaccination

67 programs. Since 2009, mass dog sterilization and vaccination programs contributed to a decline in the 
incidence of canine rabies [6]. However, the disease remains endemic in South Bhutan and in some pockets in East Bhutan. All 13 human deaths due to rabies recorded between 2009 and 2017 came from southern districts where the estimated average annual incidence is 0.4 deaths $/ 100,000$ population [7, 8]. Rabies PEP (wound treatment, vaccination with or without immunoglobulin administration) is

72 provided free of charge for the public by the government of Bhutan. Recent estimates show that current

73 PEP intervention effectively averts about 15 human deaths annually in rabies endemic areas [9].

74 However, PEP prescription without a thorough rabies exposure risk assessment results in substantial

75 costs to the health sector [10]. Between 2009 and 2016, the annual number of dog bite patients

76 increased from 1000 to over 7000 [7]. As per the Ministry of Health record, an estimated annual cost of

77 PEP in Bhutan is about Nu. 8.5 million (USD 131,000) - approximately 6\% of the essential medicines

78 budget (S1)

79 To assess the rabies risk in potentially exposed patients, the Ministry of Health in Bhutan recommends

80 clinicians follow the National Rabies Management Guidelines (NRMG) [11]. The guidelines are in line

81 with WHO recommendations. The rabies risk assessment in endemic areas should be based on the

82 nature of exposure to animals or their products and the nature of the injury. Prescription of anti-rabies

83 vaccine (ARV) is recommended for patients with a moderate or severe risk (NRMG Categories 2 and 3),

84 while ARV associated with rabies immunoglobulin (RIG) are recommended for Category 3 exposures.

85 However, a shortage of RIG supply in Bhutan resulted in RIG treatment being reserved for patients with

86 exposure to suspected or confirmed rabid animals only. The current wording in the NRMG around RIG

87 prescription lacks clarity and does not provide clear direction for clinicians.

89 rabies and rising PEP expenditure could be results of inappropriate rabies PEP prescription by clinicians.

90 However, there is currently no published evidence supporting this assumption. Therefore, this study

91 investigated rabies risk assessment and PEP prescription practices in potentially exposed patients by 
92 clinicians in the high-rabies-risk areas of southern Bhutan. The aim was to provide a better

93 understanding of clinician's knowledge and practices with respect to managing patients potentially

94 exposed to rabies and identify measures to improve these where necessary to strengthen the national

95 effort to reduce the burden of rabies.

\section{Materials and Methods}

97 We conducted a cross-sectional study to assess clinicians' rabies risk assessment process and PEP

98 prescription decisions during the management of patients potentially exposed to rabies. The study was

99 approved by the Research Ethics Board of Health, vide approval ref.no. REBH/Approval/2016/001.

\section{Study sites and participants}

101 All 13 health centers with doctors in the medical staff, i.e. hospitals and grade I Basic Health Units (BHU-

102 I), located in the high rabies-risk belt of southern Bhutan were included in the study (Fig 1). All clinicians

103 involved in the management of patients potentially exposed to rabies infection from animals were

104 included. The term 'clinician' in this study refers to staff who treat patients, including doctors and

105 paramedical staff (clinical officers and health assistants).

106 Fig 1. Map of Bhutan showing rabies high-risk region and the location of the 13 study sites (Map 107 created using open source QGIS 2.12 software downloaded from http://monde-

108 geospatial.com/download-qgis-2-12-lyon-the-last-released-version-of-qgis/ and shapes files

109 from https://gadm.org/download_country_v3.html)

\section{Study design and data collection}

112 The study was conducted from $1^{\text {st }}$ February to $31^{\text {st }}$ March 2016 . Cases were defined as all patients

113 presenting to a hospital or BHU-I for treatment following potential exposure to rabies from an animal or

114 animal product. Cases were identified prospectively and a questionnaire was completed by designated,

115 specifically trained staff in each study site, while observing the patient's consultation with the clinician. 
116 Additional information or verification was sourced directly from the clinician or from the patient where

117 adequate information was not collected by the clinician during the consultation. For example, the

118 patient was interviewed about the exposure event after the consultation if the clinician did not elicit the

119 required information. The data collected included demographic information about the patient,

120 clinicians' level of qualification and years of experience, whether the clinician used a pre-determined set

121 of epidemiological questions about the nature of the animal exposure, the patient's response, the rabies

122 risk as assessed by the clinician, and whether PEP was prescribed. Information was cross-validated from

123 copies of the PEP case record sheets maintained by hospital staff.

\section{Data analysis}

\section{Demographics of clinicians and patients}

127 Summary statistics were calculated to describe the population of clinicians performing clinical

128 assessments of patients potentially exposed to rabies, and the demographics of the patient population

129 included in the study. Given individual clinicians managed a varying number of cases potentially exposed

130 to rabies, summary statistics for clinician demographics were weighted according to the number of such

131 cases managed by each clinician. All personal identifiable information of both patient and clinician were

132 removed to anonymize and protect their privacy before the analysis of data was conducted

\section{Types of exposure}

134 The different types of potential exposure and the animal species involved were determined from each

135 patient's account of the exposure event. Exact binomial tests were used to test for equi-probability of

136 males versus females for various types of exposure.

\section{Rabies risk assessment conducted by clinicians}


Our questionnaire comprised a set of 23 epidemiological questions to evaluate the level of rabies risk in

139 a patient exposed to a potentially infected animal. The questionnaire was prepared using the NRMG and

140 a rabies expert panel and covered date, type of exposure, animal species involved, vaccination status

141 (dog and cat), potential rabies status of the animal and past PEP history of the patient.

142 We identified sets of relevant epidemiological questions for three types of exposures, namely: direct

143 exposure to an owned animal or a stray animal, and indirect exposure to any animal through contact

144 with animal products or fomites. For example, questions about indirect exposure were irrelevant for

145 direct exposure cases. Similarly, questions about vaccination status of the animal were irrelevant for

146 patients bitten by a stray animal.

147 Firstly, we described the proportion of clinicians asking the relevant epidemiological questions

148 pertaining to cases with each type of exposure. Secondly, we independently classified each case into

149 one of three rabies risk categories (none, moderate, severe) by comparing the epidemiological

150 information provided by the patient, either during or following the consultation, with the current NRMG

151 [11] . The criteria that we used for rabies risk classification are presented in Table 1.

Table 1. Criteria for rabies risk assessment and recommended PEP prescription in case of exposure to "suspect or rabid animals", extracted from the WHO adapted National Rabies 154 Management Guidelines (2014) in Bhutan

\begin{tabular}{|c|c|c|}
\hline Exposure type & Risk category & Recommended PEP \\
\hline $\begin{array}{l}\text { Licks on intact skin, touching, feeding of } \\
\text { animals. } \\
{ }^{1} \text { Consumption of butter, curd, cheese, } \\
\text { whey (dachu), cooked meat. } \\
\text { Petting, bathing or coming in contact } \\
\text { with ustensils used on a suspected rabid } \\
\text { animal. }\end{array}$ & None (Category 1) & $\begin{array}{l}\text { Not recommended, if reliable case } \\
\text { history available. }\end{array}$ \\
\hline $\begin{array}{l}\text { Person consuming unboiled or } \\
\text { unpasteurized milk, buttermilk, }\end{array}$ & $\begin{array}{l}\text { Moderate } \\
\text { (Category 2) }\end{array}$ & Wound management \\
\hline
\end{tabular}




\begin{tabular}{|l|l|l|}
\hline $\begin{array}{l}\text { uncooked meat from rabid animal. } \\
\text { Nibbling of uncovered skin by suspected } \\
\text { rabid animal. }\end{array}$ & $\begin{array}{l}\text { Provide anti-rabies vaccine immediately } \\
\text { Stop vaccination if animal remains } \\
\text { healthy throughout the observation } \\
\text { beriod of 10 days or if the animal is } \\
\text { pleeding. } \\
\text { Person who handles or prepares meat or } \\
\text { handles carcass of rabid animals. } \\
\text { reliable laboratory using an appropriate } \\
\text { diagnostic technique }\end{array}$ \\
\hline $\begin{array}{l}\text { Single or multiple transdermal bites or } \\
\text { scratches } \\
\text { Licks on broken skin } \\
\begin{array}{l}\text { Contamination with mucous membrane } \\
\text { with saliva (i.e. licks or splash on oral } \\
\text { cavity, eyes, nose, external genitalia) }\end{array}\end{array}$ & 3) & Severe (Category \\
\hline
\end{tabular}

For patients for whom the clinician assessed the rabies risk, we estimated the agreement between the

157 risk category assigned by clinicians and the independently assessed risk based on the NRMG, using the weighted Cohen's kappa statistic for ordinal variables and equal weights. Thirdly, we performed logistic regression to evaluate potential factors associated with "agreement" between the clinician and the

160 NRMG risk assessment. A binary variable "agreement" (0: disagreement between clinician and the 161 NRMG, 1: agreement between clinician and the NRMG) was created. Variables evaluated in the bivariate 162 model were:

163 - gender;

164 - professional experience (number of years);

165 - designation of the clinician: medical doctor, assistant clinical officer, health assistant;

166 - highest level of qualification: Bachelor of Medicine, Bachelor of Surgery (MBBS), Diploma or

$167 \quad$ Certificate;

168 - health center type: Basic Health Unit, district hospital, regional referral hospital;

169 - district (9 levels); 
- actual level of risk of the exposure event according to the NRMG (none, moderate, severe).

171 Variables significant at $\mathrm{P}<0.3$ were used to fit a multivariate model. Observations were clustered by

172 clinician, hospital and district, hence these 3 variables were used as nested random effects in the model.

173 We used a stepwise backward model selection process using the lowest Akaike Information Criterion

174 (AIC). Potential interactions between fixed effect variables in the final model were evaluated and

175 selected using the lowest AIC.

\section{Assessment of PEP prescription by the clinician}

177 We described the clinicians' practices in relation to the prescription of PEP, in particular ARV and RIG, 178 according to rabies risk classification as assessed by clinicians and independently assessed according to 179 the NRMG. All analyses were performed using R [12].

\section{Results}

181 Fifty clinicians participated in this study and questionnaires were completed for 273 patients. Each

182 patient had only one consultation for potential rabies exposure during the study period and each

183 clinician saw an average of 5.5 such patients (median: four patients, range 1-19). Most consultations

184 occurred in district hospitals (177/273, 64.8\%), 59 in BHU-I and 37 in regional hospitals.

\section{Clinicians' demographics}

186 All clinician level statistics were weighted by the number of consultations provided by each clinician.

187 The median age of clinicians was 31 years (range 25 to 55 years) and median duration of clinical

188 experience was 6 years (range 1 to 35 years). Doctors had a median of two years clinical experience

189 while clinical officers and health assistants had a median of 22 and 17 years, respectively. Health

190 assistants or clinical officers who held a certificate or diploma qualification conducted the majority of 
191

192

193

194

195

196

197

198

199

200

201

202

203

204

205

206

207

208

consultations (55\%) while MBBS doctors conducted the rest. Male staff performed nearly three quarters of the consultations (Table 2).

Table 2. Demographic features of clinicians who led consultations for $\mathbf{2 7 3}$ patients potentially exposed to rabies in high risk areas in Bhutan during the study period.

\begin{tabular}{lcc}
\hline Variable & Freq. & Percent. \\
\hline Designation & & \\
\hline Medical Doctor & 124 & 45.4 \\
\hline Clinical Officer (or Assistant CO) & 34 & 12.5 \\
\hline Health Assistant & 115 & 42.1 \\
\hline Gender & \\
\hline Males & 201 & 73.6 \\
\hline Female & 72 & 26.4 \\
\hline Type of Health Center & & \\
\hline District Hospitals & 177 & 64.8 \\
\hline Regional Hospitals & 37 & 13.6 \\
\hline Basic Health Units & 59 & 21.6 \\
\hline
\end{tabular}

\section{Patient demographics}

Patients' age spanned 2 to 85 years, with half under 18 years of age (137/273). The frequency of potential rabies exposure in children decreased regularly with age after 2 years of age; children $<2$ years of age were infrequently exposed (Fig 2). Of the patients presenting, 97\% were Bhutanese nationals; $55 \%$ were male, and $45 \%$ were female $(P>0.05)$. Half of the patients were preschoolers or students, and $16 \%$ were farmers (Fig 3). The majority of patients $(208 / 267,78 \%)$ presented to the health center or hospital on the day of exposure or the following day.

Fig 2. Age distribution of patients under 18 years old $(n=137)$ seeking treatment for potential exposure to rabies in the study centers during the period 1st February to 31st March 2016

Fig 3. Occupation of $\mathbf{2 7 3}$ patients presenting for treatment following potential exposure to rabies in high-risk areas in southern Bhutan 
Types of exposure

211 The majority of patients were potentially exposed to rabies as a result of dog bites $(189 / 273,69 \%)$.

212 Among dog bites, 67 (35\%) were inflicted by free-roaming (also referred to as 'stray') dogs and 123

$213(65 \%)$ by pet dogs. There was no significant difference between the proportions of male or female

214 patients for each of the exposure categories presented in Table 3.

Table 3. Animal species involved, type of exposure and demographics for $\mathbf{2 7 3}$ patients potentially exposed to rabies through contact with animals

\begin{tabular}{lcccc}
\hline Variable & Frequency (\%) & \multicolumn{3}{c}{ Patient demographics } \\
& & Male & Female & Mean age \\
\hline Animal species & & & & \\
\hline Pet dog & $140(51.3 \%)$ & 79 & 61 & 23.3 \\
\hline Free roaming dog & $81(29.7 \%)$ & 48 & 33 & 20.5 \\
\hline Cat & $40(14.7 \%)$ & 16 & 24 & 22.4 \\
\hline Cattle/buffalo & $12(4.4 \%)$ & 5 & 7 & 34.8 \\
\hline Rodents/wild animals & $5(1.8 \%)$ & 2 & 3 & 26 \\
\hline Exposure types & & & & \\
\hline Bites (with bleeding) & $152(55.7 \%)$ & 85 & 67 & 23.7 \\
\hline Bites (no bleeding) & $61(22.3 \%)$ & 35 & 26 & 22.9 \\
\hline Scratches & $52(19.1 \%)$ & 26 & 26 & 17.3 \\
\hline Licks or Nibbles & $11(4 \%)$ & 5 & 6 & 18.3 \\
\hline Carcass handling (cattle/buffalo) & $6(2.2 \%)$ & 3 & 3 & 38.2 \\
\hline $\begin{array}{l}\text { Indirect exposure (consumption of milk/milk } \\
\text { products or contact with animal products) }\end{array}$ & $8(2.9 \%)$ & 3 & 5 & 26.0 \\
\hline
\end{tabular}

\section{Rabies risk assessments}

219 In nearly all consultations, the clinicians investigated the type of rabies exposure by asking relevant

220 questions during the consultation. The exact type of exposure, the date, the wound site and the species

221 involved were obtained in over $95 \%$ of clinical assessments. Details of the epidemiological information

222 that clinicians collected to assess rabies risk are detailed in (Fig 4). The risk profile of the 272 patients for 
whom information was available to independently classify rabies risk according to the NRMG was $57 \%$

224 severe risk, $43 \%$ moderate risk and only 1 (0.3\%) was no risk.

Fig 4. Proportion of relevant epidemiological questions asked by the clinicians for each exposure type (indicated in brackets); the denominator varied between 8 and 273, depending on the frequency of the type of exposure

231 The attending clinicians recorded the corresponding rabies risk assessment (none, moderate, severe) on

232 the patient's sheet for 194 (71\%) of the 273 patients. Of these 194 risk assessments performed by

233 clinicians, only 102 (53\%) were correctly classified when compared to the NRMG (Table 4). It was more

234 frequent for clinicians to underestimate rabies risk (37\%) than to overestimate it (11\%). Of the 154 cases

235 independently classified as severe rabies risk, 62 (40\%) were correctly classified by clinicians, 45 (29\%)

236 were classified as moderate risk, 9 (6\%) were classified as having no rabies risk and 38 (25\%) were not

237 classified or recorded by the clinicians. Based on results in Table 4, the kappa agreement test statistics

238 was $0.203(p<0.001)$, indicating a poor agreement of rabies risk categorization between the clinicians

239 and the NRMG.

240 Table 4. Comparison of clinicians' classification of rabies risk versus National Rabies

241 Management Guidelines (NRMG) for $272^{1}$ patients potentially exposed to rabies

\begin{tabular}{c|cccc}
\hline & \multicolumn{5}{|c}{ Risk category assigned by the clinician: } \\
& None & Moderate & Severe & NA $^{2}$ \\
\hline Risk category (NRMG): & 0 & 1 & 0 & 0 \\
None & 17 & 40 & 20 & 40 \\
Moderate & 9 & 45 & 62 & 38 \\
Severe &
\end{tabular}

${ }^{2}$ Not Available: risk either not assessed or not recorded by the clinician 
Potential explanatory variables associated with the agreement between the clinician's risk assessment

246 and the NRMG (final model) are presented in Table 5. The effect of clinical designation interacted with

247 that of gender. Male health assistants were the most likely to make an accurate risk assessment and

248 female health assistants were the least likely. Female and male doctors were not significantly different.

249 Male health assistants were three times more likely to make an accurate risk assessment than male

250 doctors, whereas female doctors were twice as likely to be accurate than female health assistants. Male

251 health assistants were 12 times more likely to make an accurate risk assessment than female health

252 assistants. Clinicians from district or regional hospitals were much more likely to conduct accurate risk

253 assessments compared to clinicians in Basic Health Units (Odds Ratio of 7.8 and 17.6, respectively),

254 independent of the clinician's designation in the different healthcare facilities. The random effects

255 (clinicians nested in hospitals nested in districts) suggested that after taking into account the variation in

256 assessment accuracy associated with clinicians, hospitals and the fixed effects, there was no residual

257 variation between districts.

Table 5. Final multivariate logistic regression model of factors associated with making an accurate rabies exposure risk assessment, defined by agreement with the NRMG.

\begin{tabular}{lcccc}
\hline Variable & $\begin{array}{c}\text { Coefficient } \\
\text { Estimate }\end{array}$ & SE & Multiplier ${ }^{1}$ & P-value \\
\hline Gender & & & & \\
\hline Male & & & & \\
\hline Female & -0.77 & 0.55 & 0.5 & 0.16 \\
\hline Designation & & & & \\
\hline Medical Doctor & & & & \\
\hline Clinical Officer & -0.70 & 0.63 & 0.5 & 0.26 \\
\hline Health Assistant & 1.10 & 0.49 & 3.0 & 0.02 \\
\hline Health Centre Type & & & & \\
\hline Basic Health Unit & & & & 0.00 \\
\hline District Hospital & 2.05 & 0.73 & 7.8 & 0.01 \\
\hline Regional Hospital & 2.87 & 1.16 & 17.6 & 0.04 \\
\hline Female*Health Assistants ${ }^{2}$ & -1.70 & 0.84 & 0.2 & \\
\hline
\end{tabular}



compared to the reference stratum. This corresponds to the odds ratio only for variables for which there were no interactions in the model.

${ }^{2}$ The interaction between Gender and Designation; there were no female Clinical Officers in the study.

The number of patients for whom clinicians prescribed ARV by rabies risk category as assessed by the clinician and as independently assessed according to the NRMG is shown in Table 6. Neither ARV nor RIG

271 was prescribed for 1 patient assessed by the clinician to be severe risk and ARV was not prescribed for a

272 second patient assessed to be moderate risk. Conversely, clinicians prescribed ARV for 10 (38\%) of 26

273 patients whom they assessed as having no rabies risk and 75 (95\%) of 79 patients for whom they did not

274 assess rabies risk. Considering the independent risk categorization of patients according to NRGM, 7

$275(5 \%)$ of 154 severe risk and $16(14 \%)$ of 117 moderate risk patients were not prescribed ARV. PEP and

276 other treatments prescribed by the clinicians in this study are described in (Fig 5).

277 Table 6. Number of patients prescribed ARV by risk category of potential rabies exposure as 278 assessed by clinicians and as independently assessed according to the NRMG.

\begin{tabular}{c|cccc||ccc}
\hline & \multicolumn{3}{|c||}{ According to the clinician: } & \multicolumn{3}{c}{ According to NRGM: } \\
Rabies risk: & None & Mod. & Severe & NA & None & Mod. & Severe \\
\hline ARV prescription: & & & & & & & \\
No ARV & 16 & 2 & 1 & 4 & 0 & 16 & 7 \\
ARV & 10 & 84 & 81 & 75 & 1 & 101 & 147 \\
Additional RIG* & 0 & 1 & 2 & 0 & 0 & 1 & 2 \\
\hline
\end{tabular}




\section{Discussion}

287 This study was the first attempt to describe and evaluate clinical practices in the management of 288 patients potentially exposed to rabies through contact with animals, in high-rabies-risk areas of Bhutan.

289 We described and analyzed consultations for 273 patients who were potentially exposed to rabies, 290 which were conducted by 50 clinicians in 13 health centers in the study area. Most consultations were 291 conducted by health assistants and clinical officers (55\%), and the remainder by medical doctors (45\%), 292 mostly in district hospitals (65\%). Most consultations were provided by junior health workers, with a 293 median clinical experience of six years.

294 Dog bites constituted the main source of potential rabies exposure (69\%) and half of the patients were 295 under 18 years old. The frequency of potential rabies exposure in children decreased with age, except 296 for children under two years old, which were rarely presented. However, the age distribution of the 297 underlying study population was not taken into account, neither was the possible differential bias of 298 reporting to health centers following potential rabies exposure in adults versus children. Age-specific 299 risks thus cannot be inferred from these data.

300 We attempted to minimize information biases from the clinicians through prior communication.

301 Observational biases from the interviewer were mitigated by training on information recording.

302 However, clinicians could have been influenced towards more rigorous risk assessment during the study, 303 so the results likely over-estimate the performance of clinicians.

\section{$304 \quad$ Risk classification}


The independent rabies risk classification using the NRMG guidelines for 272 of the 273 cases for whom

306 information was available showed $57 \%$ of cases had a severe risk, $43 \%$ a moderate risk and only 1 case

307 was classified as having no risk. Clinicians collected epidemiological information about the type of

308 exposure and species involved for almost all cases. However, a rabies risk assessment was performed

309 and recorded for only 194 (71\%) of cases. Clinicians' risk categorization for this group showed a low level

310 of agreement with the NRMG (kappa $=0.203$ ), and a tendency for clinicians to underestimate exposure

311 risk.

312 Male health assistants were most likely to make an accurate risk assessment and female health

313 assistants were the least likely. Female and male doctors were not significantly different. Male Health

314 Assistants were more likely to accurately assess risk than male doctors (Odds Ratio=3), whereas female

315 doctors were more likely to be accurate than female Health Assistants (Odds Ratio =1.9). In addition,

316 male Health Assistants were more likely to assess rabies exposure risk according to the guidelines than

317 female Health Assistants (Odds Ratio = 12). Clinicians from district or regional hospitals were more likely

318 to conduct risk assessments in agreement with the NRMG than clinicians in Basic Health Units (Odds

319 Ratios of 7.8 and 17.6 , respectively).

320 These results possibly reflect the availability of opportunities and participation by clinicians in capacity

321 building training programs for rabies and PEP conducted by the Ministry of Health as was observed in a

322 Haitian study [13]. The attitude of clinicians towards training might differ depending on their level of

323 qualification. Health assistants are more readily available to attend such locally organized trainings.

324 Most doctors, however, are unable to attend or oblivious to district training sessions. The apparent

325 better performances of male health assistants compared to male doctors may reflect the successful

326 impact of such continuing education opportunities, rather than initial education level. The lower level of

327 agreement between risk assessments conducted by female Health Assistants and the NRMG than those

328 conducted by male Health Assistants may reflect lower access or uptake of professional training by 
women compared to their male counterparts. In addition, male health assistants were more

330 experienced (median of 29 years of clinical experience) compared to male or female doctors (median of

3312 years of experience) and female health assistants (median of 10 years of experience). Due to this

332 correlation between clinical designation and the number of years of experience, both variables could

333 not be in the final model and the best fitting model was the model with clinical designation and without

334 clinical experience.

Better performance of clinicians in hospitals compared to basic health units might be associated with lack of training and awareness of the guidelines by BHU level staff. This might also partially reflect the

337 effect of clinical experience, even though the coefficients for type of health center was virtually

338 unchanged when adding this variable to the model, after accounting for designation. Nevertheless, 339 junior clinicians are often posted in lower level health facilities rather than hospitals, as per government 340 policies. Moreover, the majority of rabies assessments (78\%) occurred in hospitals rather than basic 341 health units, hence junior clinicians in basic health units may be comparatively less experienced.

342 Specific rabies risk assessment and PEP training should target all clinicians involved in managing cases 343 potentially exposed to rabies, including doctors, since the latter is equally in the frontline and tend to 344 not perform as well as trained Health Assistants (particularly males), similar to Indian study conducted in 345 eight cities [14]. Gender parity in the training of health professionals should also be pursued to ensure 346 the engagement of female clinicians, as well as targeted training of staff in basic health units, as this is 347 likely to make an important difference in improving the accuracy of rabies assessments.

348 According to the NRMG, a rabies risk assessment is recommended in cases exposed to "suspected or 349 confirmed rabid animals". However, there is no clear definition for a "suspected" rabies case in animals. 350 In fact, since rabies is considered endemic in southern Bhutan, all potential vector animals involved in an 351 exposure event should be considered as suspected rabies and followed by a risk assessment in a health 
352 facility. Our result indicated, by contrast, that only $71 \%$ of clinicians actually performed and documented

353 a rabies risk assessment. The findings of this study suggest that risk assessment by clinicians and PEP

354 decisions was mostly based on the type of exposure (i.e. bite, bite with puncture wound, licks, nibbles,

355 indirect exposure) which are clearly outlined in the national guidelines (Table 1). However, they tend to

356 misclassify the risk based on the patient's answer. It is a concern that $13 \%$ of patients were mis-classified

357 as having no risk, while they had moderate or severe risk.

358 Irrespective of the risk assessment, the vast majority of patients (91.6\%) still received ARV treatment

359 even when the risk was not assessed. Clinicians in high-risk areas of Bhutan thus proved relatively

360 conservative in their attitude towards PEP prescription. However, for the 23 patients in the study who

361 did not receive ARV, 16 were misclassified as having no rabies risk category, including three patients that

362 were in fact in the severe risk category. Conversely, the only patient with no risk of rabies was still

363 prescribed ARV, and another in the category of moderate risk was unduly prescribed RIG. This is similar

364 to findings of a nationwide study conducted between 2005 and 2008, which reported frequent PEP

365 administration in category I exposures [10]. The discrepancy between clinician's practice and the

366 national guidelines in our study lies in underestimating the rabies risk in the first place, rather than a

367 lack of compliance with recommendations in subsequent PEP prescription. As a result, seven patients

$368(2.6 \%)$ in the highest risk category had not received the appropriate treatment (neither ARV nor RIG). In

369 this study, eight patients were exposed to "confirmed rabies" cases, all of which were cows (the

370 exposure consisting of handling the carcass and drinking raw milk). Another 76 patients were exposed to

371 animals classified as "suspected of rabies" by the clinician, including 62 dog bites. However, RIG was

372 prescribed to only 3 patients (1\%) which is in concurrence with the results of the earlier study that RIG

373 was not regularly administered to dog bite victims in Bhutan [10]. Two of the patients receiving RIG

374 were bitten by dogs suspected of rabies, the third after drinking milk and handling the carcass of a

375 confirmed rabid cow. The risk for this patient was mis-classified by the clinician from moderate 
376 (according to the guidelines) to high. This highlights the need to raise the awareness of clinicians to

377 more frequently prescribe RIG for patients bitten by suspected rabid animals, as the situation in this

378 respect does not seem to have changed since 2011 [10]. In Bhutan, the availability of RIG is very limited,

379 as in many rabies endemic countries [15]. Hence, the national guidelines contain modified

380 recommendations indicating RIG for only most severe category 3 exposure, i.e. exposure to "suspected

381 or confirmed" rabid cases. However, these terms are not clearly defined in the guidelines. For dog bites,

382 discrepant advice co-exists to take into account extra-severity criteria, such as free-roaming versus pet

383 dog, or vaccination status. Improving the supply in RIG treatment and clarifying the guidelines would

384 help to improve the decisions on RIG by clinicians.

385 Clinical experience and good clinical judgment are essential to prevent human rabies [16]. Other studies

386 on clinician's knowledge and attitudes, conducted in the USA (Florida, Kentucky), showed an

387 unsatisfactorily low level of compliance with national guidelines resulting in inappropriate PEP

388 treatment [17-19]. Studies in countries free of rabies [20] or in endemic areas [21-23] similarly reported

389 insufficient rabies risk assessment due to a lack of familiarity of clinicians with the recommendations,

390 and highlighted the need for knowledge updating [14]. Lack of agreement between rabies risk

391 assessment by clinicians and the guidelines was also apparent in our study, although only a low

392 percentage resulted in subsequent inappropriate prescription. In contrast, public health physicians in

393 Israel showed a very high level of compliance to PEP guidelines [24]. This could be due to different public

394 health policies or better training or expertise of clinicians undertaking rabies assessment in Israel

395 compared to clinicians in Bhutan. Moreover, access to immunoglobulin may not limit the compliance

396 with the recommendations in Israel.

397 From a resource allocation standpoint, greater compliance with guidelines was shown to increase the 398 benefit-cost ratio of PEP use in other studies $[25,26]$. A study in a low rabies-risk area (Massachusetts, 399 USA) highlighted how large amounts of rabies PEP could be wasted in patients with low or non-existent 
risk of rabies exposure [27]. By contrast, our study in high risk area showed that due to a tendency to

401 underestimate the rabies risk, patients with moderate or severe risk were sometimes not prescribed

402 PEP. A similar study in low rabies-risk areas of Bhutan would potentially shed light on potential

403 differences in PEP decision patterns and whether unnecessary treatments occur more frequently in

404 those areas.

405 This study highlighted important gaps in clinical practice in the management of patients exposed to

406 rabies risk from animals in high-rabies-risk districts in Bhutan. Further progress in preventing human

407 rabies deaths could be achieved by improving the clinical decisions in the event of animal exposure,

408 while rationalizing the use of ARV and RIG. One key strategy is an ongoing education of clinicians to

409 improve the accuracy of rabies risk assessment. An update of the national guidelines should accompany

410 the training of professionals. In particular, improving clarity about epidemiological criteria for RIG

411 prescription would help to prioritize patients needing RIG treatment. RIG administration in high-risk

412 areas of the country, where category 3 exposure is frequent, is currently very low. In line with current

413 WHO recommendation, optimizing access and uptake of RIG by animal bite victims through use of

414 modified recommendations at the local level is strongly recommended including integrated

415 management of animal bites patients in rabies endemic areas [4]. Further studies in low-rabies-risk

416 areas of Bhutan are required for a full assessment of the situation regarding attitudes and practices of

417 clinicians towards rabies in this country.

\section{Acknowledgments}

419 We thank the following organization and the staffs whose contributions made this study possible.

420 The Chief Medical Officer and focal health staff in 13 hospitals under Ministry of Health, Royal

421 Government of Bhutan. The study team also is grateful to staff at the Faculty of Nursing and Public 
Health (FNPH), Khesar Gyalpo University of Medical Sciences of Bhutan and staff at the College of

423 Natural Resources (CNR), Royal University of Bhutan.

\section{References}

427 1. World Health Organization. WHO Expert Consultation on Rabies. Second report. Geneva: World 428 Health Organization; 2013. Report No.: 9241209828.

$4292 . \quad$ Gongal G, Wright AE. Human Rabies in the WHO Southeast Asia Region: Forward Steps for 430 Elimination. Adv Prev Med. 2011;2011:383870.

$431 \quad 3 . \quad$ Karunanayake D, Matsumoto T, Wimalaratne O, Nanayakkara S, Perera D, Nishizono A, et al. 432 Twelve years of rabies surveillance in Sri Lanka, 1999-2010. PLoS Negl Trop Dis. 2014;8(10):e3205.

433 4. World Health Organization. WHO expert consultation on rabies: third report. Geneva: World 434 Health Organization; 2018.

435 5. Tenzin T, Wangchuk S, Dorji T, SMckenzie J, DJolly P. Dog bites and Human Rabies:

436 Epidemiological Analysis of post exposure prophylaxis in Bhutan (2009-2012). Bhutan Journal of animal 437 science. 2017:52.

4386 Tenzin, Wangdi K, Ward MP. Human and animal rabies prevention and control cost in Bhutan, 439 2001-2008: the cost-benefit of dog rabies elimination. Vaccine. 2012;31(1):260-70.

$4407 . \quad$ Ministry of Health. Annual Health bulletin. Thimphu; 2017 [Available from:

441 http://www.health.gov.bt/wp-content/uploads/ftps/annual-health-

442 bulletins/Annual\%20Health\%20Bulletin-2017.pdf

443 8. National Statistics Bureau. Statistical Yearbook of Bhutan 2017. 37th ed. Thimphu

444 BhutanSeptember 2017 [Available from: www.nsb.gov.bt/publication/files/pub9ot4338yv.pdf

445 9. Tenzin, Dhand NK, Gyeltshen T, Firestone S, Zangmo C, Dema C, et al. Dog bites in humans and 446 estimating human rabies mortality in rabies endemic areas of Bhutan. PLoS neglected tropical diseases. 447 2011;5(11):e1391.

448 10. Tenzin, Dhand NK, Ward MP. Human rabies post exposure prophylaxis in Bhutan, 2005-2008:

449 Trends and risk factors. Vaccine. 2011;29(24):4094-101.

450 11. Ministry of Health. National Guideline for Management of Rabies 2nd Edition ed.

451 Thimphu:Bhutan 2014 [Available from: http://www.health.gov.bt/wp-content/uploads/moh-

452 files/2015/07/National-Guideline-for-Management-of-Rabies-2nd-Edition-2014.pdf

453 12. R RCT. A language and environment for statistical computing. R Foundation for Statistical 454 Computing, Vienna, Austria. 2014 [Available from: http://www.R-project.org/.

455 13. Fenelon N, Dely P, Katz MA, Schaad ND, Dismer A, Moran D, et al. Knowledge, attitudes and 456 practices regarding rabies risk in community members and healthcare professionals: Petionville, Haiti, 457 2013. Epidemiol Infect. 2017;145(8):1624-34.

458 14. H.R. Ravish, B. Gangaboraiah, H.A.N. Doddabele, A. Rachana, V.Veena. Knowledge, attitude, and 459 practice of rabies prophylaxis among physicians at Indian animal bite clinics. Asian Biomedicine. April $460 \quad$ 2013;7(2):237-42. 
15. Wilde H, Lumlertdacha B, Meslin FX, Ghai S, Hemachudha T. Worldwide rabies deaths prevention-A focus on the current inadequacies in postexposure prophylaxis of animal bite victims. Vaccine. 2016;34(2):187-9.

464 16. Tepsumethanon S, Tepsumethanon V, Tantawichien T, Suwansrinon K, Wilde H. Problems in human rabies post-exposure prophylaxis management. Travel Med Infect Dis. 2007;5(3):189-93. 17. Conti L, Wiersma S, Hopkins R. Evaluation of state-provided postexposure prophylaxis against rabies in Florida. South Med J. 2002;95(2):225-30.

18. Auslander M, Kaelin C. Rabies Postexposure Prophylaxis Survey-Kentucky, 1994. Emerging Infectious Diseases. 1997;3(2):199.

470 19. Moore DA, Sischo WM, Hunter A, Miles T. Animal bite epidemiology and surveillance for rabies 471 postexposure prophylaxis. JAVMA. 2000;217(2).

472 20. Jeanpetit R, Bellanger AP, Piotte E, Haffner-Mauvais C, Marguet P. Knowledge, attitudes and practices of primary care physicians in the Franche-Comte region (France) regarding the risk of rabies. Zoonoses Public Health. 2014;61(5):371-6.

21. Nayak RK, Walvekar PR, Mallapur MD. Knowledge, Attitudes and Practices regarding Rabies among general practitioners of Belgaum City. 2013;6(3):237-42.

477 22. Koruk ST, Koruk I, Kutlu S. Where Do We Stand in the Control of Rabies? Knowledge and 478 Practices Among Physicians in a Health District in Turkey. WILDERNESS \& ENVIRONMENTAL MEDICINE. 479 2011;22:151-55.

480 23. Ramesh Holla, Bhagawan Darshan, Astha Guliani, Bhaskaran Unnikrishnan, Rekha Thapar, 481 Prasanna Mithra, et al. How familiar are our doctors towards Rabies prophylaxis- A study from coastal 482 south India. PLoS Negl Trop Dis. 2017;11(10).

483 24. Dubnov J, Hefer E, Rubin L, Rishpon S. A change in rabies post-exposure treatment guidelines 484 after decision analysis in Israel. Eur J Public Health. 2007;17(1):92-7.

485 25. Ribadeau Dumas F, N'Diaye DS, Paireau J, Gautret P, Bourhy H, Le Pen C, et al. Cost-effectiveness of rabies post-exposure prophylaxis in the context of very low rabies risk: A decision-tree model based on the experience of France. Vaccine. 2015;33(20):2367-78.

488 26. Moran GJ, Talan DA, Mower W, Newdow M, Ong S, Nakase JY, et al. Appropriateness of rabies 489 postexposure prophylaxis treatment for animal exposures. Emergency ID Net Study Group. JAMA.

$490 \quad$ 2000;284(8):1001-7.

491 27. Kreindel S, McGuill M, Rupprecht C, DeMaria A. Rabies postexposure prophylaxis: When is it 492 appropriate? Infectious Diseases in Clinical Practice. 1998;7(6):274-9.

\section{Supporting files}




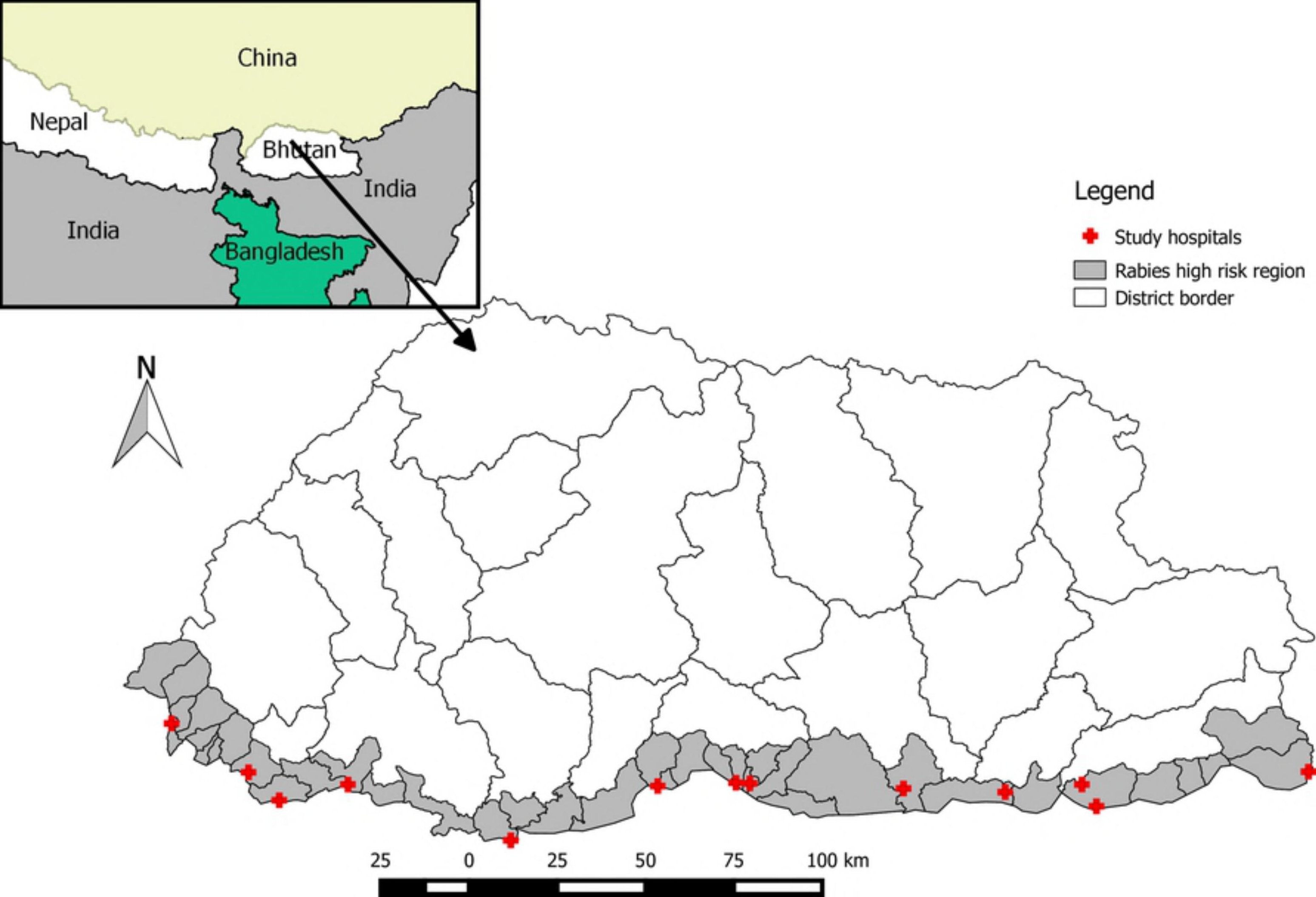


Students -

Pre-school-

Private Sector-

Farmers -

43

Other-

Public Services -

34

31
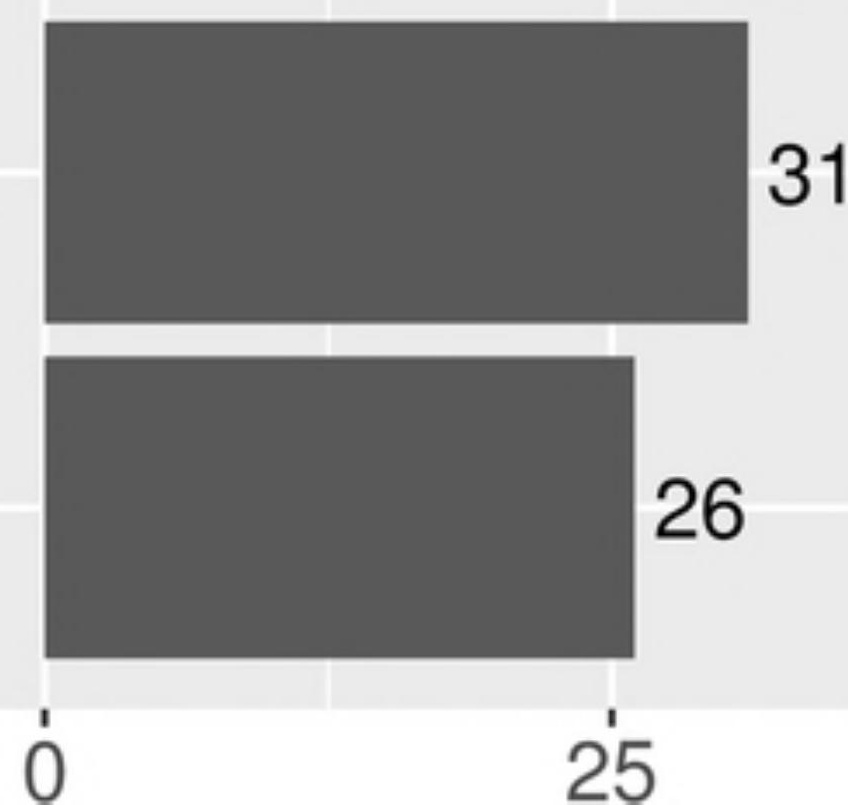

50

75 
Type of indirect exposure (for indirect expo.) -

Type of exposure (direct vs.indirect) Type of direct exposure (for direct expo.) Date of exposure Species involvedWound site Duration animal observation (if available) Animal vaccination status (for pet) Wound washedBite/scratch provoked Animal available observation (for dog/cat) Animal abnormal behaviour Animall suspect or confirmedReceived prior PEP Other people bitten (for bites) Antiseptic application by the patient-

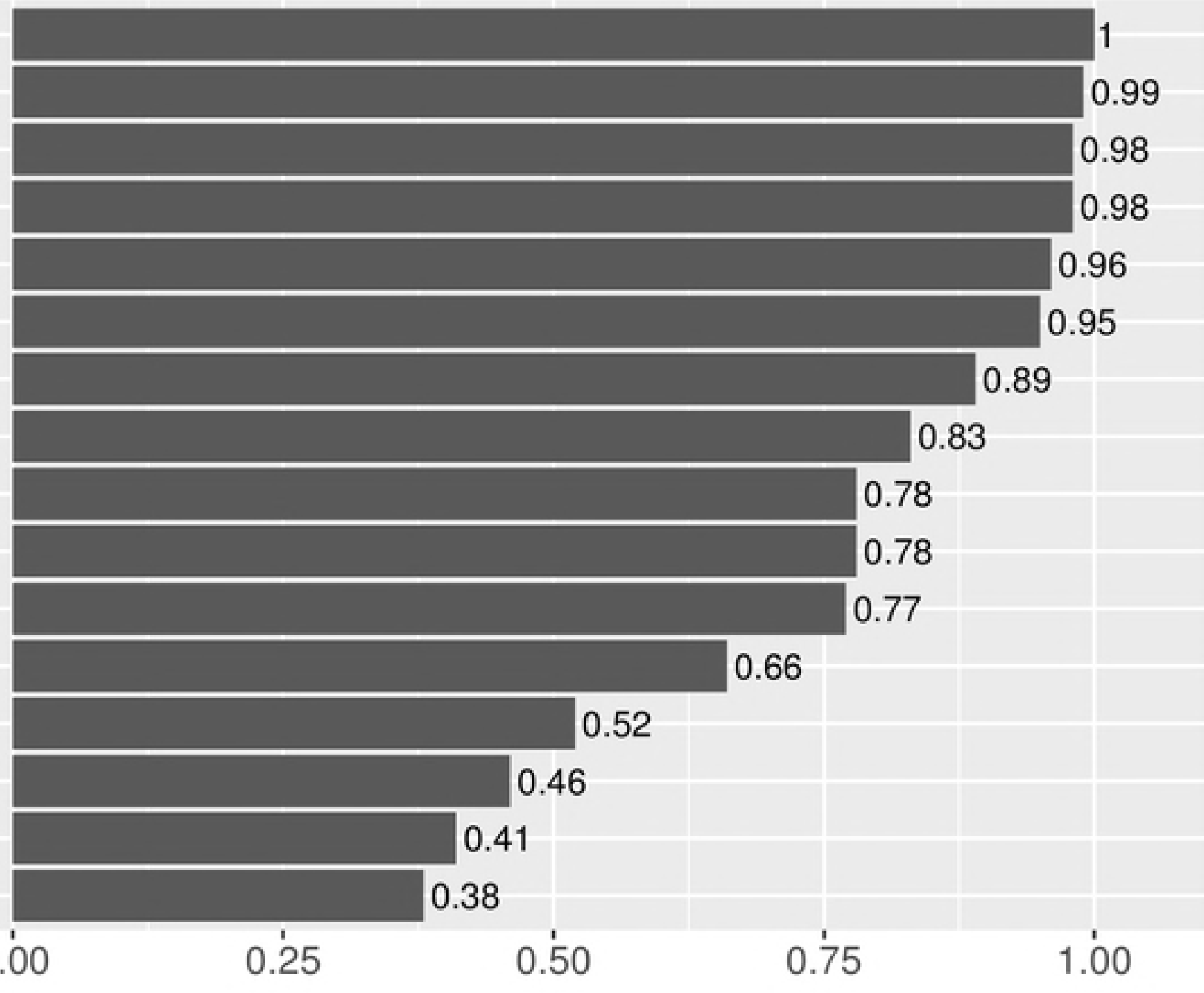

Proportion of assesments with clinicians asking relevant epidemiological questions 
ARV intra-dermal-

Counselling Wash wound with soap -

Tetanus toxoid Antiseptic Wound dressing Reassurance Antibiotics -

Other treatment -
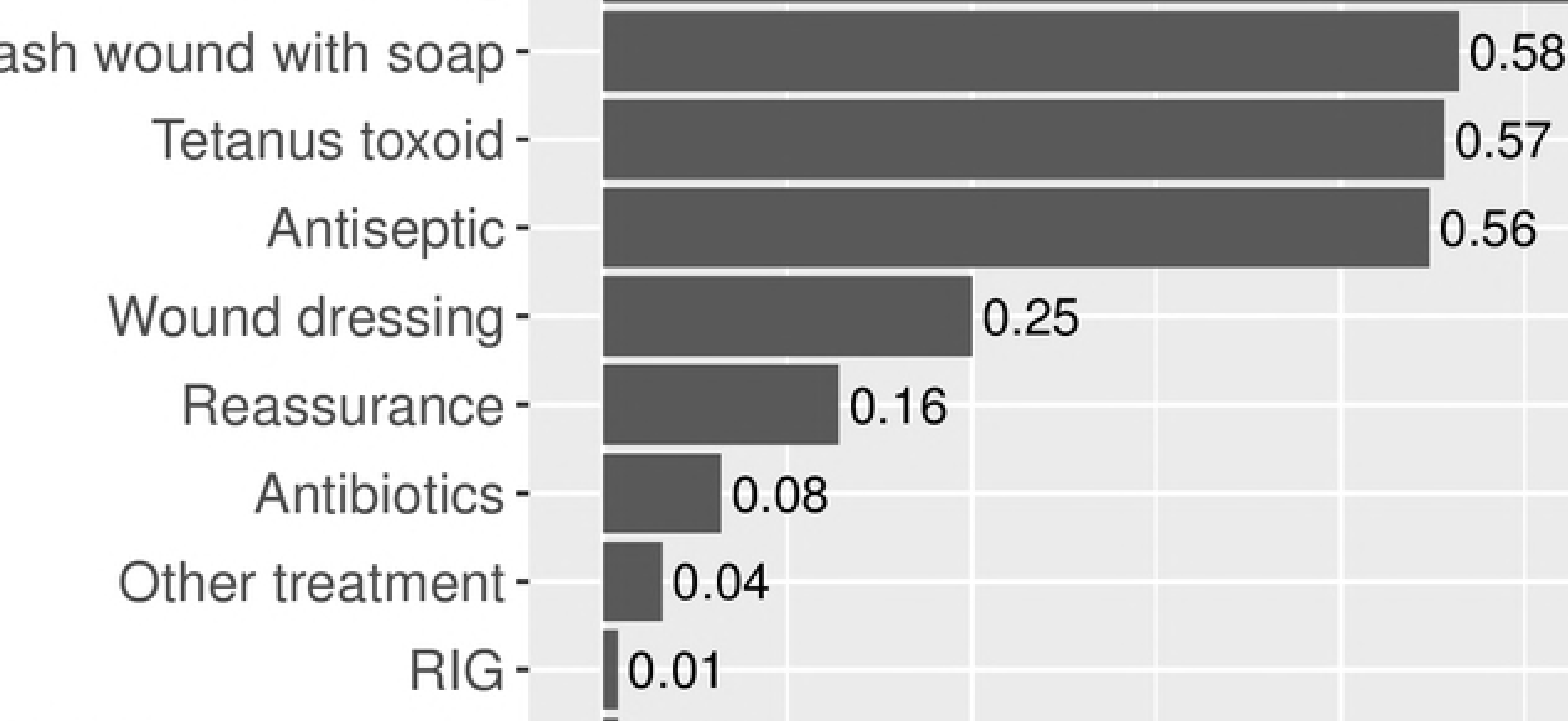

$\begin{array}{ll}\text { ARV intra-muscular - } & 0.01\end{array}$

Wound suturing -
0.00
0.25
0.50
0.75
1.00
Proportion of assesments with different treatments prescribed 